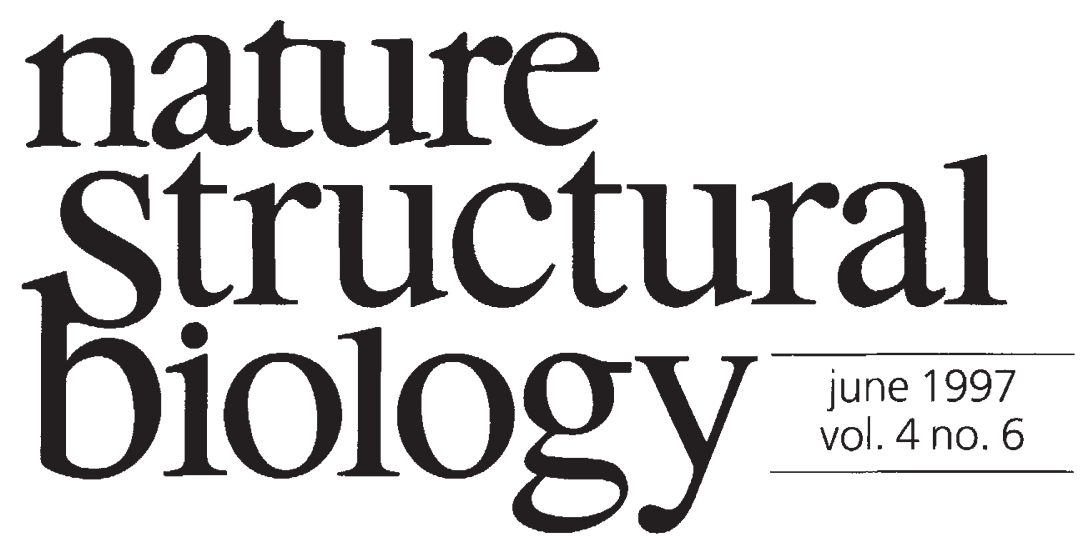

\title{
Antibiotics and RNA
}

Blocking protein synthesis is an effective way of stopping an organism dead in its tracks and many antibiotics function in just this manner. Of course, evolution often provides a means of side-stepping such frontal assaults; the structure of the rRNA methyltransferase ErmAM from Streptococcus pneumoniae reported by Fesik and colleagues in this issue ${ }^{1}$ illustrates one of the ways that bacterial pathogens can dodge the lethal effects of antibiotics, while at the same time potentially revealing a bacterial Achilles' heel.

The structural and functional differences in protein synthesis machinery across the prokaryotic/eukaryotic divide makes the complex set of chemical reactions orchestrated by the ribosome all-the-more attractive as a target for a molecular 'spanner in the works'. Many of the most effective antibiotics are made by fungi (erythromycin, for example): the selectivity for prokaryotes_and the ability to take the compounds in high doses without undue toxicity in humans_-has made, and makes such antibiotics extremely useful in clinical practice.

Of course, under the kind of selection pressure provided by sub-lethal dosing regimes, prokaryotic pathogens have evolved resistance to many anti-bacterials, including the macrolide (erythromycin, spiramycin), lincosamide (lincomycin) and streptogramin type B antibiotics (vernamycin B), collectively known as the MLS antibiotics. Three modes of resistance have been noted for erythromycin, and the MLS antibiotics in general: target-site modification, inactivation and efflux.

By far the most common mechanism of resistance is target-site modification, and in particular the methylation of a specific adenine base in the 23S ribosomal RNA. This modification-involving the mono- or dimethylation of the N6 amino group of adenine - is carried out by a family of rRNA methyltransferases, known collectively as the erythromycin ribosomal methlyase (Erm) family, which use S-adenosylmethionine as the methyl donor. The methylation of this adenine base interferes with the binding of the antibiotics to the prokaryotic ribosome, abrogating their ability to interfere with protein synthesis.

Methylation of certain genomic sequences is also used by bacteria to distinguish their own DNA from that of invading viruses as part of their restriction/modification systems. Indeed, the first structure of such a methyltransferase was that of a DNA modifying enzyme, Hhal DNA methyltransferase ${ }^{2}$. Although lacking significant sequence similarity, the larger of the two domains of ErmAM is strikingly similar to 


\section{editorial}

\section{IMAGE \\ UNAVAILABLE FOR COPYRIGHT REASONS}

the catalytic domains of the other methyltransferases whose structures have been reported ${ }^{2-7}$. The smaller, substrate-binding domain of ErmAM is, on the other hand, strikingly different.

\section{RNA binding motifs}

Of the methyltransferases with known structure, only two bind RNA; ErmAM and vaccinia virus VP397. Even so, the structures of their substrate binding domains, which consist mainly of $\alpha$-helices, bare little resemblance to each other (and all the other structurally characterised methyltransferases), and little similarity to other RNA binding proteins whose structures are known (Fig. 1a, $b$ ).

The three most common of the RNA binding modules--ribonucleoprotein modules, double-stranded RNA binding domains and K-homologous domains-all have an $\alpha / \beta$-type fold similar to that found in many ribosomal proteins. For proteins containing such domains RNA is generally found to bind

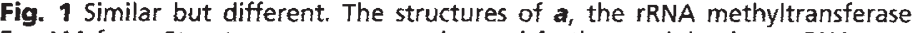
Streptococcus pneumoniae and $\boldsymbol{b}$, the vaccinia virus mRNA specific 2'-O-methyltransferase (and vaccinia poly(A) polymerase processivity factor) VP39. ErmAM is composed of two domains; the lower, catalytic dor the side of the structure. (Figure from ref. 1).
1. Yu, L. et al. Nature Struct. Biol. 4, 483-489 (1997).

2. Cheng, $X$., Kumar, S., Posfai, J., Pflugrath, J.W. \& Roberts, R.J. Cell 74, 299-307 (1993).

3. Vidgren, J., Svensson, L.A. \& Liljas, A. Nature 368, 354-358 (1994).

4. Labahn, J.et al. Proc. Natl. Acad. Sci. USA 91 10957-10961 (1994).

5. Reinisch, K.M., Chen, L., Verdine, G.L. \& Lipscomb, W.N. Cel/ 82, 143-153 (1995)

6. Fu, Z. et al. Biochemistry 35, 11985-11993 (1996)

7. Hodel, A.E., Gershon, P.D., Shi, X. \& Quiocho, F.A. Cel/ 85, 247-356 (1996).

8. Hodel, A.E. et al. Nature Struct. Biol. 4 350-354 (1997).

9. Berglund, H., Rak, A., Serganov, A. Garber M. \& Härd, T. Nature Struct. Biol. 4, 20-23 (1997).

10. Xing, Y., GuhaThakurta, D. \& Draper, D.E. Nature Struct. Biol. 4, 24-27 (1997).

11. Markus, M.A., Hinck, A.P., Huang, S., Draper, D.E. \& Torchia, D.A. Nature Struct. Biol. 4, 70-77 (1997).

12. Yonath, A. \& Franceschi, F. Nature Struct. Biol. 4, 3-5 (1997). to the surface of the $\beta$-sheets, with the bases of the RNA stacking onto aromatic side chains protruding from the face of the sheet. Clearly, ErmAM and VP39 must interact with their substrates in a different manner.

VP39, as well as serving as a processivity factor for the vaccinia poly(A) polymerase, is an mRNA cap-specific 2'-O-methyltransferase. The enzyme is composed of a single domain, with the consensus catalytic core-the $\beta$-sheet of which is almost completely buried within the protein-being adorned with a number of additional C-terminal $\alpha$-helices which, together with the loops connecting the elements of secondary structure, constitute essentially the entire surface of the protein (but do not form a distinct, separate domain; Fig. $1 b$ ).

Although little is known about the mode of mRNA binding (which is required for the capping activity) for VP39, the location of the cap-binding site has been identified $^{8}$. It seems most likely that the capped mRNA stretches across the active-site baring face of VP39 (see Fig. $3 a$, d in ref. 2) whereas it is suggested that the surface of the backside of the protein (composed in part of C-terminal $\alpha 7, \alpha 8$ and $\alpha 9$ ) may play a role in RNA binding in VP39's role as a processivity factor. Certainly, this region of the protein seems to have features indicative of an involvement in binding RNA.

The putative rRNA binding domain of ErmAM is composed of four $\alpha$-helices (Fig. 1a). A casual glance might suggest that the domain is reminiscent of the rRNA binding domains recently identified in the proteins $S 15$ and $\mathrm{L}_{1}{ }^{9-12}$. Closer examination indications that any resemblance is superficial, though, and limited mainly to their $\alpha$-helical composition: S15 and L11 have a helix-turn-helix motif which can be aligned with the homeodomain motif seen in some DNA binding proteins. It is likely that ErmAM binds a fairly large region of the $23 \mathrm{~S}$ rRNA, indicating that a fairly extensive portion of the protein may be involved in the interaction. Indeed, one entire face of the protein is lined with positive charged residues, which may form part of this RNA binding site.

One way to defeat such resistance mechanisms is to disable them, rather than sidestepping them by developing drugs against different target sites. The structure of ErmAM opens up the possibility of designing drugs that block the resistance conferring methylation activity of the protein. Such drugs could then be coadministered with MLS antibiotics, once again rendering them effective against previously resistant strains of bacteria. 